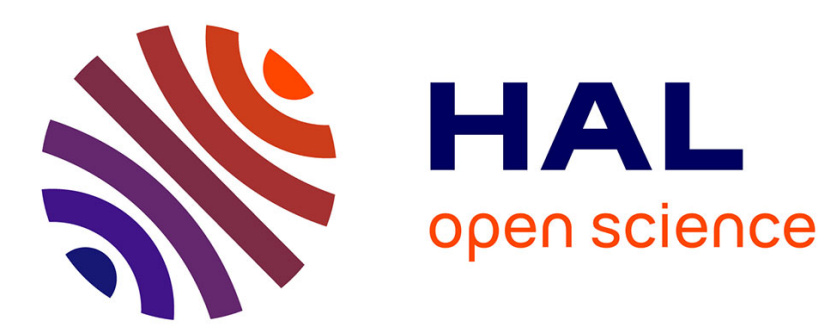

\title{
A replica analysis of the travelling salesman problem
}

\author{
M. Mézard, G. Parisi
}

\section{To cite this version:}

M. Mézard, G. Parisi. A replica analysis of the travelling salesman problem. Journal de Physique, 1986, 47 (8), pp.1285-1296. 10.1051/jphys:019860047080128500 . jpa-00210320

\section{HAL Id: jpa-00210320 https://hal.science/jpa-00210320}

Submitted on 1 Jan 1986

HAL is a multi-disciplinary open access archive for the deposit and dissemination of scientific research documents, whether they are published or not. The documents may come from teaching and research institutions in France or abroad, or from public or private research centers.
L'archive ouverte pluridisciplinaire HAL, est destinée au dépôt et à la diffusion de documents scientifiques de niveau recherche, publiés ou non, émanant des établissements d'enseignement et de recherche français ou étrangers, des laboratoires publics ou privés. 
Classification

Physics Abstracts

$05.20-75.50-85.40$

\title{
A replica analysis of the travelling salesman problem
}

\author{
M. Mézard (*) and G. Parisi (**) \\ Dipartimento di Fisica, Universita' di Roma I, Piazzale Aldo Moro 2, 00185 Roma, Italy
}

(Reçu le 27 janvier, accepté le 23 avril 1986)

\begin{abstract}
Résumé. - Nous proposons et analysons une solution symétrique dans les répliques des problèmes de voyageurs de commerce à liens indépendants. Elle fournit des estimations analytiques raisonnables pour les quantités thermodynamiques comme la longueur du chemin le plus court.
\end{abstract}

Abstract. - We propose and analyse a replica symmetric solution for random link travelling salesman problems. This gives reasonable analytical estimates for thermodynamic quantities such as the length of the shortest path.

\section{Introduction.}

In trying to understand the theory of spin glasses, a number of methods and concepts have been developed which seem to have possible applications beyond statistical mechanics, particularly in the field of combinatorial optimization.

It was soon realized [1] and demonstrated [2] that the determination of the ground state of an infinite range spin glass is an NP complete problem. As this is probably the NP complete problem which has been most studied by physicists, let us briefly recall the kind of results that can be obtained in this case. The infinite range Ising spin glass is a set of $N$ spins $\sigma_{i}= \pm 1$ interacting through random couplings $J_{i j}$ which are independent Gaussian random variables with $\overline{J_{i j}}=0$ and $\overline{J_{i j}^{2}}=1 / N$. The $J$ 's are quenched variables given once for all (a given sample, i.e. a set of $J$ 's, is an instance of the problem), and the problem consists in finding the ground state configuration of the spins, that is the one which minimizes the Hamiltonian

$$
H=-\sum_{1 \leqslant i<j \leqslant N} J_{i j} \sigma_{i} \sigma_{j}
$$

NP completeness means that there is no known algorithm which can solve every instance of this problem in a time growing less than a power of $N$, and it is unlikely that such an algorithm can be

(*) On leave from Laboratoire de Physique Théorique de l'ENS, Paris.

(**) Universita' di Roma II, Tor Vergata, and INFN, Sezione di Roma. found [3]. A good heuristic (an algorithm which provides an approximate solution) is the simulated annealing method which consists in sampling the configurations with a Boltzmann-Gibbs probability $\mathrm{e}^{-\boldsymbol{H} / \boldsymbol{T}}$ with the Monte Carlo method, and smoothly decreasing the temperature [4]. This is a general and powerful approach for many complex optimization problems [4-7].

On the other hand some kind of analytical information is also available. The ground state energy density $E / N$ (minimum of $H / N$ ) converges to a certain value $E_{0}$ for large $N$, independently of the sample $\left({ }^{1}\right)$.

The replica method allows one to prove that there is a phase transition in this system at the critical temperature $T_{c}=1$. Below $T_{c}$ there is a breaking of ergodicity and one needs replica symmetry breaking. A scheme for such a breaking was proposed in [8], which allows us to compute $E_{0}$ precisely

$$
E_{0}=-0.7633 \pm 0.0001 \text {. }
$$

This kind of prediction is essentially probabilistic (for large $N$ the value of $E / N$ for a given sample is a Gaussian random variable of mean $E_{0}$ and width $\left.\overline{\left(\frac{E}{N}-E_{0}\right)^{2}} \sim \frac{1}{N}\right)$, but it can be of direct interest, for instance for testing heuristics.

Although other relevant information can be obtained from the replica solution of this model (e.g. the

( $\left.{ }^{1}\right)$ This property of " self averageness " is obtained with the replica method and well established numerically, but has not yet been demonstrated rigorously. 
ultrametric topology of the space of equilibrium states below $T_{c}$ [9] and the correlations of the spin values in these states [10]), no algorithm has been found yet which can use these informations in order to speed the search for the ground state. Naturally the simulated annealing algorithm is the one which takes the greatest advantage of the analytical results of thermodynamics : once one knows the critical temperature $T_{\mathrm{c}}$ (or a freezing temperature in the case where there is no sharp phase transition), one must perform a very slow annealing around $T_{c}$, while the regions far above $T_{c}$ (where the system thermalizes rapidly) or far below $T_{\mathrm{c}}$ (where the system is frozen) can be swept more rapidly.

In this paper we want to show how the replica method can be used generally to get the same kind of information as described above on other NP complete problems. We have chosen to apply it to the travelling salesman problem mainly for aesthetic reasons (it is among the easiest NP complete problems to state, and probably the most studied), and also because some numerical data were previously available. It is also a problem rather far from the spin-glass one. This will exemplify one power of the replica method : the fact that no a priori knowledge of the nature of the order and of the order parameter is required.

This method has already been used to predict the ground state energy in the matching problem [11] (a polynomial problem) and in the bipartitioning of infinitely connected random graphs which was shown to be equivalent to the S.K. [12].

In section 2 we describe the problem and its statistical mechanics representation.

In section 3 we propose a replica symmetric solution and analyse it. It is the most technical part of this paper and can be skipped by the reader who does not appreciate the beauty of replica computations.

The results are presented and discussed in section 4.

\section{Statistical mechanics of the travelling salesman problem.}

The travelling salesman problem(TSP) is the following. Given $N$ points and the distances $d_{i j}$ between any two of them, find the shortest tour, i.e. closed connected path, that goes through all the points.

A popular family. of TSP's is the one where $d_{i j}$ 's are Euclidean distances between points distributed randomly in a square. The resulting correlations between the distances allow for very powerful heuristics (for instance a combination of simulated annealing and "divide and conqueer" strategies [4-6]), but they are difficult to take into account in the replica method.

In the more general case the $d_{i j}$ represent complicated costs which do not possess Euclidean correlations. We shall study a family in which good numerical results are far more difficult to achieve than in the Euclidean case, the one where $d_{i j}$ 's are independent random variables with the same distribution $\rho(d)$ [7-13] (we keep to the symmetric case $d_{i j}=d_{j i}$ ). In this case the low temperature properties, and particularly the length of the shortest tour, depend only on the behaviour of $\rho(d)$ at small $d$. If :

$$
\rho(d)^{d} \vec{\sim}^{0} d^{r} / r !
$$

the nearest neighbour of any point $i$ is at a distance $d \sim N^{-\frac{1}{r+1}}$ and one can show [13] that the length of the shortest tour is of order $N \times N^{-\frac{1}{r+1}}$. A tricky case is $r=0$ in which the best known upper bound on the shortest tour is of the order of $\log N$. This case has also been extensively studied numerically by Kirkpatrick and Toulouse [7], and for these reasons our numerical results in the next sections will be given in this case. We predict a finite length of the shortest path, and not a $\log N$ growth.

In order to extract from the $\frac{(N-1) !}{2}$ tours those which contribute, it was shown in [13] that the partition function which is naturally introduced in the statistical mechanics description of optimization problems must be of the form :

$$
Z_{\mathrm{TSP}}=\sum_{\text {tours }} \exp \left(-\beta N^{\frac{1}{r+1}} L_{\text {tour }}\right)
$$

where $\beta$ is an inverse "temperature». The energy density $E=-\frac{1}{N} \frac{\partial \log Z_{\mathrm{TSP}}}{\partial \beta}$ is $N^{-1+\frac{1}{r+1}}$ times the average length of the tours at the temperature $T=1 / \beta$.

The whole difficulty of the TSP lies in the fact that the sum in (2.2) is over tours, i.e. closed connected paths. This constraint of connectivity is highly nonlocal and difficult to implement with local variables. We use a procedure that has been invented in the theory of polymers [14] and introduce on each site $i$ an $m$-component spin $S_{i}$ of fixed length $S_{i}^{2}=m$. As was shown by Orland [15], one is then led to study a generalized partition function :

$$
\begin{gathered}
Z=\int \prod_{i} \mathrm{~d} \mu\left(\mathbf{S}_{i}\right) \exp \left(\gamma \sum_{i<j} \mathrm{e}^{-\beta N \frac{1}{r+1}} d_{i j} \mathbf{S}_{i} \cdot \mathbf{S}_{j}\right) \\
\mathrm{d} \mu(\mathbf{S}) \equiv C_{m} d^{m} \mathbf{s} \delta\left(\mathbf{S}^{2}-m\right)\left(\int \mathrm{d} \mu(\mathbf{S})=1\right)
\end{gathered}
$$

which reduces to $Z_{\mathrm{TSP}}$ in the limit $m \rightarrow 0, \gamma \rightarrow \infty$ [15] :

$$
Z_{\mathrm{TSP}}=\lim _{m \rightarrow 0} \frac{1}{m} \lim _{\gamma \rightarrow \infty} \frac{Z}{\gamma^{N}}
$$

as can be shown using the special simplifications of integrals over $\mathbf{s}$ in the limit $m \rightarrow 0$, summarized by the formula :

$$
\int \mathrm{d} \mu(\mathbf{S}) \mathrm{e}^{\mathrm{h} . \mathbf{S}} \stackrel{m \rightarrow 0}{\sim} 1+\frac{h^{2}}{2} .
$$


As we explained in the introduction, extensive thermodynamic quantities such as the free energy $F=-\frac{1}{\beta} \log Z_{\text {TSP }}$ are self averaging, which means that $F / N$ goes for $N \rightarrow \infty$ towards a limit which is sample independent. Hence we shall try to compute the average $\overline{\log Z_{\mathrm{TSP}}}$, using the replica method which consists in computing $\overline{Z_{\mathrm{TSP}}^{n}}$ and using :

$$
\overline{\log Z_{\mathrm{TSP}}}=\lim _{n \rightarrow 0}\left(\overline{Z_{\mathrm{TSP}}^{n}}-1\right) / n .
$$

From (2.4) one must compute $\overline{Z^{n}}$ which is equal to :

$$
\overline{Z^{n}}=\int \prod_{i=1}^{N} \prod_{a=1}^{n} \mathrm{~d} \mu\left(\mathbf{S}_{i}^{a}\right) \prod_{i<j} \exp \left[\gamma \mathrm{e}^{-\beta N \frac{1}{r+1} d_{i j}} \sum \mathbf{S}_{i, a} \cdot \mathbf{S}_{j, a}\right]
$$

where we have introduced, on each site $i, n$ replicas of the original spin variable $: S_{i}^{a}, a=1, \ldots, n$.

Because of the statistical independence of the $d_{i j}$ 's and of the introduction of replicas, the averages over each $d_{i j}$ decouple in (2.7) and using :

$$
\int_{0}^{\infty} \rho(l) \exp \left(a \mathrm{e}^{-\beta N \frac{1}{r+1} l}\right) \mathrm{d} l^{N>1} \sim+\frac{1}{N} \sum_{p=1}^{\infty} \frac{a^{p}}{p !} g_{p}
$$

where :

one finds :

$$
g_{p}=(p \beta)^{-(r+1)}
$$

$$
\overline{Z^{n}}=\int \prod_{i=1}^{N} \prod_{a=1}^{n} \mathrm{~d} \mu\left(\mathbf{S}_{i}^{a}\right) \exp \left[\frac{1}{2 N} \sum_{p=1}^{\infty} \frac{\gamma^{p} g_{p}}{p !} \sum_{\substack{a_{1}, \ldots, a_{p} \\ \alpha_{1}, \ldots, \alpha_{p}}}\left(\sum_{i} S_{i, a_{1}}^{\alpha_{1} \ldots S_{i, a_{p}}}\right)^{\alpha_{p}}\right]
$$

where the $a$ 's, going from one to $n$, are replica indices while the $\alpha$ 's, going from one to $m$, characterize the various spin components. A crucial remark is that in (2.10) the terms where at least two replica indices are equal lead to vanishing contributions in the limit of the TSP (2.4) (which must be taken before the $n \rightarrow 0$ limit) as is shown in appendix I. A Gaussian transformation decouples the various sites in (2.10), leading to :

$$
\begin{aligned}
\overline{Z_{\mathrm{TSP}}^{n}}=\lim _{m \rightarrow 0}\left(1 / m^{n}\right) \lim _{\gamma \rightarrow \infty}\left(1 / \gamma^{n N}\right) \int \prod_{p=1}^{n} \prod_{a_{1}<\ldots<a_{p}} \prod_{\alpha_{1} \ldots \alpha_{p}} \mathrm{~d} Q_{a_{1} \ldots a_{p}}^{\alpha_{1} \ldots \alpha_{p}} \times \\
\times \exp -N\left[\frac{1}{2} \sum_{p=1}^{n} \frac{1}{g_{p}} \sum_{a_{1}<\ldots<a_{p}} \sum_{\alpha_{1} \ldots \alpha_{p}}\left(Q_{a_{1} \ldots \alpha_{p}}^{\alpha_{1} \ldots \alpha_{p}}\right)^{2}-\log z\right]
\end{aligned}
$$

where $z$ is the one-site partition function :

$$
z=\int \prod_{a=1}^{n} \mathrm{~d} \mu\left(\mathbf{S}_{a}\right) \exp \left[\sum_{p=1}^{n} \gamma^{p / 2} \sum_{a_{1}<\ldots<a_{p}} \sum_{a_{1} \ldots \alpha_{p}} Q_{a_{1} \ldots a_{p}}^{\alpha_{1} \ldots \alpha_{p}} S_{a_{1}}^{\alpha_{1}} \ldots S_{a_{p}}^{\alpha_{p}}\right] .
$$

In principle, formulae (2.11) and (2.12) give the free energy of the TSP in the large $N$ limit through a saddle point evaluation of the integral in (2.11). However it is well known from the theory of spin glasses that this saddle point is not easy to find in the limit $n \rightarrow 0$. Furthermore there are two other complications here : the appearance of all the order parameters $Q_{a}, Q_{a b}, \ldots, Q_{1 \ldots n}$ (similar to the case of the matching [11]), and the presence of a polymeric index $\alpha$ going from one to $m$, where $m \rightarrow 0$. We shall propose and analyse a solution of these saddle point equations in the next section.

Before going to this analysis, let us explain how other quantities, besides the thermodynamic functions, can be deduced from the saddle point values of the $Q$ 's.

An interesting information is the average distribution $P(q)$ of overlaps between the relevant tours at a given temperature. We define the overlap $q_{t, t^{\prime}}$ between two tours $t$ and $t^{\prime}$ as their number of common bonds, divided by $N$ [7]. The characteristic function $g(y)$ of $P(q)$ is defined as :

$$
g(y)=\int P(q) \mathrm{e}^{y q} \mathrm{~d} q=\sum_{t, t^{\prime}} \frac{-\beta N \frac{1}{r+1}\left(L^{t}+L^{t^{\prime}}\right)}{Z_{\mathrm{TSP}}^{2}} \mathrm{e}^{y q^{t^{\prime} t}} .
$$


In order to compute $g(y)$ within the present field - theoretical approach, we follow the same method as in spin glasses [16] and introduce two copies of a given instance - two travelling salesmen who must visit the same set of cities. This can be done by using two copies $\mathbf{S}_{i}$ and $\mathbf{S}_{i}^{\prime}$ of the $m$ component spins, with some suitable link auxiliary variables which count their number of common links. The denominator $Z_{\mathrm{TSP}}^{2}$ in $(2.13)$ can be obtained with the replica method by introducing $n-2$ independent other replicas of the system : they contribute a factor $Z_{\text {TSP }}^{n-2}$ which gives the correct result for $n \rightarrow 0$. This whole computation is described in some detail in Appendix II. The final result for $P(q)$ is :

$$
P(q)=\lim _{n \rightarrow 0} \frac{1}{n(n-1)} \sum_{a \neq b} \delta\left(q-\frac{1}{2} \sum_{p=2}^{\infty} \frac{1}{(p-2) ! g_{p}} \sum_{\substack{\alpha, \beta, \alpha_{3}, \ldots, \alpha_{p} \\ a_{3}, \ldots, a_{p} \\ \neq a, b}}{ }^{\prime}\left(Q_{a b a_{3} \ldots a_{p}}^{\alpha \beta \alpha_{3} \ldots \alpha_{p}}\right)^{2}\right)
$$

where the $Q_{a_{1} \ldots a_{p}}^{\alpha_{1} \ldots \alpha_{p}}$ takes its saddle point value 1 his result is very similar to that already found in the matching problem, the only difference being the presence of vector spin indices.

Here also, one can define generalized overlaps between $k>2$ tours as $1 / N$ times the number of bonds common to all of them. Their distribution $P^{(k)}(q)$ is then :

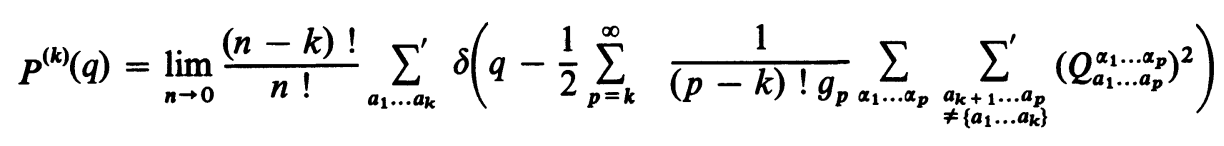

where the $\sum_{a_{k+1} \ldots a_{p} \neq\left\{a_{1} \ldots a_{k}\right\}}^{\prime}$ is the sum over all choices of the indices $a_{k+1}, \ldots, a_{p}$, each of them being distinct from all the others and from the $a_{1}, \ldots, a_{k}$.

Finally let us also describe the computation of the distribution of lengths of the links. This strictly follows the computation of the matching [11], and we include it here for completeness. The important links are those of length $\sim N^{-\frac{1}{r+1}}$, and the distribution $P(L)$ is defined as :

$$
P(L)=\frac{1}{Z_{\mathrm{TSP}}} \sum_{t} \mathrm{e}^{-\beta L_{t}} \frac{1}{N} \sum_{(i, j) \in t} \delta\left(L-d_{i j} N^{\frac{1}{r+1}}\right)
$$

where $\sum_{(i, j) \in t}$ denotes the sum over all the $N$ links $(i, j)$ which belong to the tour $t$. In order to compute the $m$ th moment $M_{m}$ of $P(L)$ we introduce a modified TSP partition function defined as :

$$
\hat{Z}_{\mathrm{TSP}}(\alpha)=\sum_{t} \exp \left(-\sum_{(i, j) \in t}\left[\beta N^{\frac{1}{r+1}} d_{i j}+\alpha N^{-1+\frac{m}{r+1}}\left(d_{i j}\right)^{m}\right]\right)
$$

so that :

$$
M_{m}=-\left.\frac{\partial \log \hat{Z}_{\mathrm{TSP}}(\alpha)}{\partial \alpha}\right|_{\alpha=0}
$$

The $\hat{Z}_{\text {TSP }}(\alpha)$ can be computed from a generalized function similar to $Z$ in (2.3), with a simple modification of the weight of each link, from $\gamma \exp \left(-\beta N^{\frac{1}{r+1}} d_{i j}\right)$ to $\gamma \exp \left(-\beta N^{\frac{1}{r+1}} d_{i j}-\alpha N^{-1+\frac{m}{r+1}} d_{i j}^{m}\right)$.

The corresponding one-link integral in (2.8) is changed into :

$$
\int_{0}^{\infty} \rho(l) \exp \left(a \exp \left[-\beta N^{\frac{1}{r+1}} d_{i j}-\alpha N^{-1+\frac{m}{r+1}} d_{i j}^{m}\right]\right) \stackrel{N \rightarrow \infty}{\sim} 1+\frac{1}{N} \sum_{p=1}^{\infty} \frac{a^{p}}{p !}(\beta p)^{-(r+1)}\left[1-p \frac{\alpha}{N}(\beta p)^{-m} \frac{(\dot{r}+m) !}{r !}\right] .
$$

So the introduction of the extra weighting proportional to $\alpha$ can be simply absorbed into a change of $g_{p}$ in (2.11) into

$$
g_{p} \rightarrow g_{p}\left[1-p \frac{\alpha}{N}(\beta p)^{-m} \frac{(r+m) !}{r !}\right]
$$


From (2.18) and (2.20), we get :

$$
\begin{aligned}
M_{m} \equiv \int P(L) L^{m} \mathrm{~d} L=\lim _{n \rightarrow 0}-\left.\frac{1}{n} \frac{\partial}{\partial \alpha} \overline{\left[\hat{Z}_{\mathrm{TSP}}(\alpha)\right]^{n}}\right|_{\alpha=0} & = \\
& =\lim _{n \rightarrow 0} \frac{1}{2 n} \sum_{p=1}^{\infty} \frac{(\beta p)^{-m}}{g_{p}} p \frac{(r+m) !}{r !} \sum_{a_{1}<\ldots<a_{p}} \sum_{\alpha_{1} \ldots \alpha_{p}}\left(Q_{a_{1} \ldots a_{p}}^{\alpha_{1} \ldots \alpha_{p}}\right)^{2}
\end{aligned}
$$

from which one obtains the distribution of lengths $P(L)$ :

$$
P(L)=\lim _{n \rightarrow 0} \frac{1}{2 n} \sum_{p=1}^{\infty}(\beta p)^{2 r+2} \frac{p L r}{r !} \mathrm{e}^{-\beta p L} \sum_{a_{1}<\ldots<a_{p}} \sum_{\alpha_{1} \ldots \alpha_{p}}\left(Q_{a_{1} \ldots a_{p}}^{\alpha_{1} \ldots \alpha_{p}}\right)^{2} .
$$

\section{The solution within a replica symmetric ansatz}

As the full set of saddle point equations is complicated, we have made some hypotheses, compatible with these equations, on the type of saddle point we look for.

As for the replica indices we make the assumption of replica symmetry :

$$
Q_{a_{1} \ldots a_{p}}^{\alpha_{1} \ldots \alpha_{p}}=Q^{\alpha_{1} \ldots \alpha_{p}}
$$

independently of the values of the $a$ 's.

The spin component indices $\alpha$ must be treated differently since the symmetry in this space is a rotational symmetry instead of the permutation symmetry of the replicas. The problem is more similar to that of vector spin glasses, but in the limit where the dimensionality $m$ of the spin goes to zero. We thus make the simple hypothesis of a spontaneous breaking of the rotational symmetry (for large values of $\gamma$ ) with :

$$
Q^{\alpha_{1} \ldots \alpha_{p}}=Q_{p} u^{\alpha_{1}} \ldots u^{\alpha_{p}}
$$

where $u$ is a given vector which we normalize to $\mathbf{u}^{2}=1$. (3.1) and (3.2) are two strong hypotheses. We shall postpone their discussion to the next section and first compute the free energy of the TSP within this ansatz.

First of all let us notice that the $m^{n}$ term necessary to insure the convergence of (2.11) in the TSP limit appears in $\bar{Z}^{n}$ from the integrals over the $Q$ 's. Because of the spontaneous breakdown of the rotational symmetry there is a Goldstone mode associated with arbitrary independent rotations in each replica :

$$
Q_{a_{1} \ldots a_{p}}^{\alpha_{1} \ldots \alpha_{p}} \rightarrow\left(\mathcal{R}_{a_{1}}\right)^{\alpha_{1} \alpha_{1}^{\prime}} \ldots\left(\mathcal{R}_{a_{p}}\right)^{\alpha_{p} \alpha_{p^{\prime}}} Q_{a_{1} \ldots a_{p}}^{\alpha_{1}^{\prime} \ldots \alpha_{p}^{\prime}}
$$

where $\mathcal{R}_{1}, \ldots, \mathcal{R}_{n}$ are rotation matrices in the $m$ dimensional space of the vectors $\mathbf{S}_{a}$. As the surface of an $m$ dimensional unit sphere vanishes linearly with $m$ for $m \rightarrow 0$, the integrals over $Q$ 's in (2.11) are proportional to $m^{n}$ for small $m$.

One must now compute the one-site partition function :

$$
z=\int \prod_{a=1}^{n} \mathrm{~d} \mu\left(\mathbf{S}_{a}\right) \exp \left[\sum_{p=1}^{n} \gamma^{p / 2} \frac{Q_{p}}{p !} \sum_{a_{1} \ldots a_{p}}^{\prime}\left(\mathbf{S}_{a_{1}} \cdot \mathbf{u}\right) \ldots\left(\mathbf{S}_{a_{p}} \cdot \mathbf{u}\right)\right]
$$

where the $\Sigma^{\prime}$ means that all the indices must be different from each other. In the TSP limit (2.4) we must pick up the term of order $\gamma^{n}$ in $z$ and take its limits $m \rightarrow 0$ and then $n \rightarrow 0$. In Appendix III we show that the result is $(\mathrm{F} 2)$ :

$$
\lim _{m \rightarrow 0} \lim _{y \rightarrow \infty} z / \gamma^{n} n \vec{\sim}^{0} 1+n \int_{0}^{\infty} \frac{\mathrm{d} \lambda}{\lambda}\left(\mathrm{e}^{-\lambda}-\int D z \exp \left[\sum_{p=1}^{\infty} \frac{Q_{p}}{p !}(i \sqrt{\lambda})^{p} H e_{p}(z)\right)\right] .
$$

From (2.11) and (3.5) we have the free energy of the TSP :

$$
\frac{1}{N} \overline{\log Z_{\mathrm{TSP}}}=-\frac{1}{2} \sum_{p=1}^{\infty} \frac{(-1)^{p-1}}{p g_{p}} Q_{p}^{2}+\int_{0}^{\infty} \frac{\mathrm{d} \lambda}{\lambda}\left(\mathrm{e}^{-\lambda}-\int D z \exp \left[\sum_{p=1}^{\infty} \frac{Q_{p}}{p !}(i \sqrt{\lambda})^{p} H e_{p}(z)\right)\right]
$$

$\left(^{2}\right) D z$ is a Gaussian integration measure and He are Hermite polynomials. These quantities are defined precisely in the appendix. 
where the $Q$ 's satisfy the saddle point equations :

$$
Q_{k}=(-1)^{k} k g_{k} \int_{0}^{\infty} \frac{\mathrm{d} \lambda}{\lambda} \int D z \int D t^{\prime} \frac{\left[\sqrt{\lambda}\left(t^{\prime}+i z\right)\right]^{k}}{k !} \exp \left[\sum_{p=1}^{\infty} \frac{Q_{p}}{p !}(i \sqrt{\lambda})^{p} H e_{p}(z)\right]
$$

One can introduce a generating function $g(x)=\sum_{k=1}^{\infty} \frac{x^{k}}{k !} Q_{k}$, and from (3.7) one gets the following integral equation for $g$ :

$$
g(x)=\int_{0}^{\infty} \frac{\mathrm{d} \lambda}{\lambda} \int D z \int D t^{\prime} \sum_{p=1}^{\infty} \frac{(-1)^{p} p g_{p}}{(p !)^{2}}\left[\sqrt{\lambda}\left(t^{\prime}+i z\right) x\right]^{p} \exp \int D t g(\sqrt{\lambda}(t+i z))
$$

This equation is rather similar to that found in the matching problem [11], apart from the complications due to Hermite polynomials and imaginary terms. Unfortunately the behaviour of the Kernel :

$$
\sum_{p=1}^{\infty} \frac{(-1)^{p} p g_{p}}{(p !)^{2}} \int D t^{\prime}\left[\sqrt{\lambda}\left(t^{\prime}+i z\right) x\right]^{p}
$$

for large values of the arguments is more complex and we have not been able to find out the correct asymptotic behaviours of $g$.

In this situation it was impossible to solve the integral equation (3.8) by a simple iterative method : we have used a different method to compute $\bar{Z}^{n}$, which consisted in a high-temperature expansion of the order parameters.

We shall present the formalism needed to build up this expansion in the general case where $g_{p}=$ $(p \beta)^{-(r+1)}$, and we shall restrict our numerical computation to the case, $r=0$. We note :

$$
b=\beta^{r+1} .
$$

In (3.7) we rescale $\lambda \rightarrow \frac{\lambda}{Q_{1}^{2}}$ and introduce the variables :

$$
X_{1}=b Q_{1}^{2} ; X_{p}=\frac{Q_{p}}{b^{p-1} Q_{1}^{p}}(p>2)
$$

which satisfy the following equations :

$$
X_{p}=\frac{(-i)^{p}}{p ! X_{1}^{p} p^{r}} \int_{0}^{\infty} \frac{\mathrm{d} \lambda}{\lambda} \lambda^{p / 2} \int D z H e_{p}(z) \mathrm{e}^{i z l \bar{\lambda}} A(z, \lambda)
$$

$X_{1}=(-i) \int_{0}^{\infty} \frac{\mathrm{d} \lambda}{\lambda} \lambda^{1 / 2} \int D z H e_{1}(z) \mathrm{e}^{i z l \bar{\lambda}} A(z, \lambda)$

with :

$$
A(z, \lambda) \equiv \exp \sum_{r=2}^{\infty} b^{r-1} X_{r} \frac{t^{r}}{r !} \lambda^{r / 2} H e_{r}(z) .
$$

The energy density is expressed in terms of these variables as :

$$
E=\frac{1}{2 \beta(r+1)}\left[X_{1}+\sum_{p=2}^{\infty}(-1)^{p-1} b^{p-1} X_{1}^{p} X_{p}^{2} p^{r}\right]
$$

Clearly in the high temperature limit $\beta \leqslant 1$ the function $A(z, \lambda)$ can be approximated by 1 , and one gets :

$$
\begin{aligned}
& X_{1}=2(1+0(b)) \\
& X_{p}=1 / p(1+0(b)) \quad p>2 .
\end{aligned}
$$

In order to compute the high temperature expansion of $2 \beta E$ up to the order $b^{N}$, one expands each $X_{i}(1<i<N)$ up to the order $b^{N+1-i}$. This can be systematically done from (3.12 to 3.14$)$ by first expanding the function $A(z, \lambda)$ to order $b^{N}$, then performing the integrals $(3.12,3.13)$ to establish the set of $(N+1)$ equations between the $X_{i}^{\prime}$ s, and finally solving these equations iteratively.

We have carried out this expansion numerically in the case $r=0$ up to the order $N=20$. The coefficients $a_{k}$ of the series

$$
2 \beta E=2+\sum_{k=0}^{19} a_{k} \beta^{k+1}
$$

are given in the first column of the table.

Although the $a_{k}$ are rational numbers we have represented them on the computer as floating point numbers. In the actual computation we have done, quadruple precision ( $\sim 30$ significant digit) has been used; from various checks we know that the error due to rounding increases exponentially with $k$ and only the first 10 digits of $a_{19}$ are correct. This accuracy is far enough for our aims; the whole computation takes a few minutes of Vax 8600. From the expansion of the $Q$ 's one can compute other interesting quantities but let us first show in the case of energy how one can use this high temperature expansion to obtain the low temperature properties of the system. It is convenient 
to perform the following manipulations. We first put aside the first two terms and write :

$$
2 E(\beta)=\frac{2}{\beta}+1+2 \hat{E}(\beta) .
$$

We then rescale $\beta$ by a factor $5^{1 / 3}$ and Borel transform this series, introducing :

$$
g(u)=\sum_{k=1}^{19} \frac{a_{k}}{k !}\left(5^{1 / 3} u\right)^{k} .
$$

So that :

$$
2 \hat{E}(\beta)=\int_{0}^{\infty} \mathrm{d} u g\left[\beta 5^{-1 / 3} u\right] \mathrm{e}^{-u} .
$$

The coefficients $\frac{a_{k} 5^{k / 3}}{k !}=b_{k}$ are given in the second column of the table. The alternance of three plus signs with three minus signs indicates the presence of complex singularities in $u$ in the three directions $(-1)^{1 / 3}$. This suggests to change variables from $u$ to $x$, where

$$
u=\frac{x}{\left(1-x^{3}\right)^{\gamma}}
$$

By trial and error, we have found that $\gamma=\frac{1}{6}$ is a good choice in the sense that the series expansion of the function

$$
f(x)=\sum_{k=1}^{19} b_{k}\left[\frac{x}{\left(1-x^{3}\right)^{\gamma}}\right]^{k}=\sum_{k=1}^{19} c_{k} x^{k}
$$

converges rather well inside the unit circle. The coefficients $c_{k}$ are given in the third column of the table.

The ground state energy (length of the shortest tour) is :

$$
\lim _{\beta \rightarrow \infty} E(\beta)=\frac{1}{2}+f(1)
$$

which can be evaluated directly from (3.23). However the successive approximants to $f(1)$ obtained by adding new terms to its series expansion still have some oscillations. A better method is to compute from (3.19)-(3.21) the function $E(\beta)$ at finite temperature. It turns out the the series is very convergent for $\beta \leqslant 2$ (the relative fluctuations in the values of $E(\beta)$ obtained from successive orders from 10 to 20 are less than $1 \%$ ), and the value of temperature $T=0.5$ is below the freezing region, so that $E(T)$ can be interpolated safely to $T=0$.

\section{Results and discussion.}

In this section we present the results obtained within the replica symmetric solution described above. As has been explained in section 3 , the high temperature expansion allows for a very precise computation of the

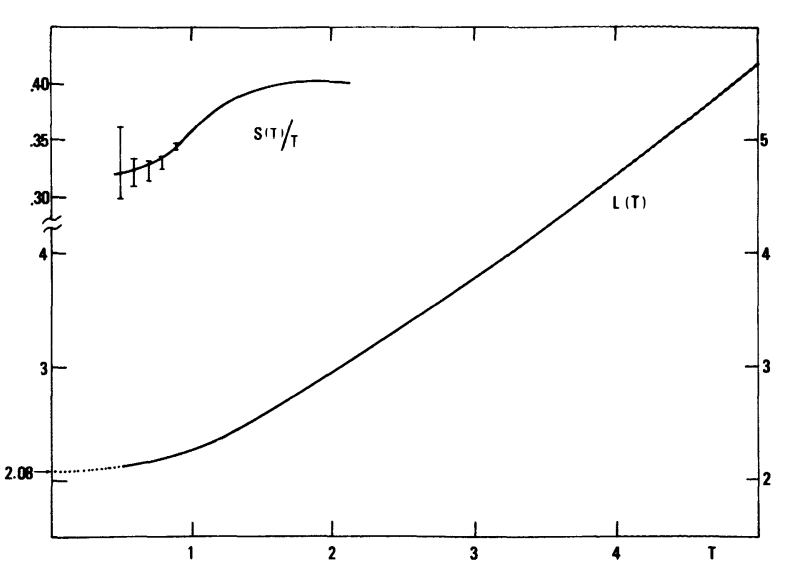

Fig. 1. - Average length $L$ as a function of the temperature $T$, for $r=0$. Note that, from (2.2), $T$ is $1 / N$ times the " usual " temperature. In the upper left corner (with the upper left scale), the entropy $S$ divided by $T$, as a function of $T$. The error bars indicate the fluctuations in the values of $S / T$ obtained from successive orders in the high temperature expansion, between the 10th and the 20th order. These fluctuations are negligible (less than $1 \%$ ) in $L(T)$ for $T>0.5$.

function $E(T)$ (average length of the tours found at a given temperature) for $T \geqslant 0.5$. The result is plotted in figure 1 . We have also computed in the same regime the entropy

$$
S(T)=\log T+\frac{\hat{E}(T)}{T}-\int_{T}^{\infty} \frac{\hat{E}(\tau)}{\tau^{2}} \mathrm{~d} \tau
$$

where $\hat{E}$ is the function introduced in (3.19).

It turns out that $\frac{S(T)}{T}$ is nearly constant in the low temperature range, with a value of the order of $0.33 \pm 05$ (see Fig. 1), the estimate of the error being quite subjective.

This suggests that the specific heat $C=T \frac{\mathrm{d} S}{\mathrm{~d} T}=\frac{\mathrm{d} E}{\mathrm{~d} T}$ grows linearly at low temperature and so the energy should start quadratically :

$$
E(T)=E(0)+\frac{1}{2}(0.33) T^{2}
$$

This allows to interpolate the curve $E(T)$ down to $T=0$. The ground state energy is :

$$
E(T=0)=2.88 \pm 0.03
$$

where the estimation of the error is again subjective.

When the temperature tends to zero the entropy converges to a value near to 0 (e.g. $0 \pm 0.03$ ), but it is of course impossible to state whether $S(0)$ is exactly zero or not.

We have also computed the distribution of overlaps between the tours from formula (2.14). Within the 
Table I. - Results for $r=0 . a_{k}$ is the coefficient of $\beta^{k-1}$ in the high temperature expansion of $2 \beta E . b_{k}=\frac{a_{k} 5^{k / 3}}{k !}$ is the coefficient after rescaling and Borel transforming. $C_{k}$ are the coefficients of the new series (3.23) obtained from $2 \hat{E}(\beta)$ after the change of variable (3.22). $d_{k}$ is the coefficient of $\beta^{k+1}$ in the high temperature expansion of $2 q_{0}$.

\begin{tabular}{rrrrr}
$k$ & $a_{k}$ & \multicolumn{1}{c}{$b_{k}$} & \multicolumn{1}{c}{$c_{k}$} & $d_{k}$ \\
\hline 0 & 1.000000 & 1.000000 & 1.000000 & 2.000000 \\
1 & 1.222222 & 2.089971 & -2.089971 & 0.666667 \\
2 & 1.750000 & 2.558516 & 2.558516 & 1.166667 \\
3 & 1.398519 & 1.165432 & 1.165432 & -6.044444 \\
4 & -4.881945 & -1.739168 & -1.390840 & -11.60833 \\
5 & -27.53473 & -3.354669 & -2.501830 & 23.01997 \\
6 & -41.35313 & -1.435872 & -0.8531563 & 263.3219 \\
7 & 263.4408 & 2.234511 & 1.278257 & 742.0547 \\
8 & 1932.493 & 3.503623 & 1.276625 & -2201.064 \\
9 & 3070.568 & 1.057708 & 0.0588726 & -29584.90 \\
10 & -34883.26 & -2.054726 & -0.2672521 & -93440.93 \\
11 & -274012.4 & -2.509022 & 0.0420933 & 425892.4 \\
12 & -384474.3 & -0.5016609 & 0.0132258 & 5825488 \\
13 & 766676.0 & 1.315821 & -0.0272207 & $1.951409 \times 10^{7}$ \\
14 & $6.287688 \times 10^{7}$ & 1.318082 & 0.116572 & $-1.204130 \times 10^{8}$ \\
15 & $7.941193 \times 10^{7}$ & 0.1897737 & 0.0524543 & $-1.723766 \times 10^{9}$ \\
16 & $-2.398438 \times 10^{9}$ & -0.6125602 & -0.0370652 & $-6.411737 \times 10^{9}$ \\
17 & $-2.132721 \times 10^{10}$ & -0.5478927 & 0.0678134 & $4.353857 \times 10^{10}$ \\
18 & $-3.177768 \times 10^{10}$ & -0.0775535 & 0.0565673 & $7.085880 \times 10^{11}$ \\
19 & $9.821437 \times 10^{11}$ & 0.2157200 & -0.0306577 & $3.200175 \times 10^{12}$
\end{tabular}

replica symmetric ansatz $P(q)$ is a delta function at a value $q_{0}$ equal to

$$
q_{0}=\frac{1}{2} \sum_{p=2}^{\infty} \frac{p-1}{g_{p}}(-1)^{p} Q_{p}^{2}
$$

The high temperature expansion of the $Q_{p}$ 's described in section 3 gives a high temperature expansion of $q_{0}$, for $r=0$ :

$$
2 q_{0}=\sum_{k=0}^{19} d_{k} \beta^{k+1}
$$

The coefficients $d_{k}$ are given in the fourth column of the table. Assuming that the analytic structure of $q_{0}(\beta)$ is essentially the same as that of $E(\beta)$. We have performed on the series $q_{0}(\beta)$ exactly the same manipulations as those we did on $E(\beta)$ in section 3. Again the curve $q_{0}(T)$ can be obtained precisely for $T=\frac{1}{\beta} \geqslant 0.6$ and is plotted in figure 2 . We have interpolated $q_{0}(T)$ linearly at low temperatures, using the property

$$
q_{0}(T)^{T} \leqslant^{1} 1-a T
$$

which is suggested by the following argument. Keeping $r=0$, the distribution of lengths of the links in the

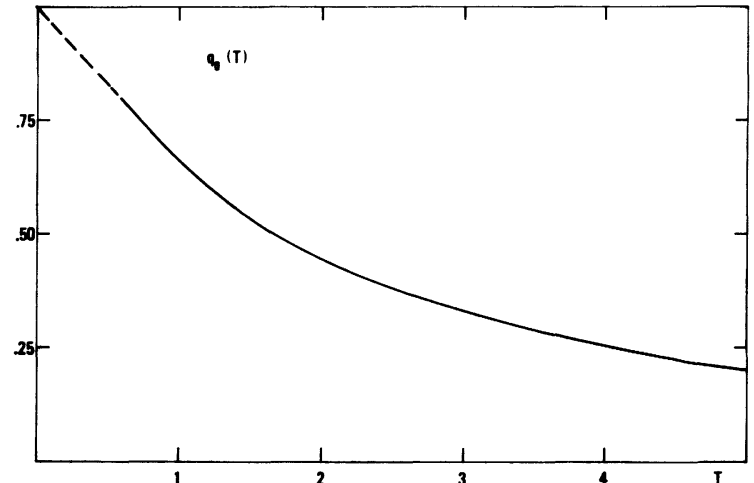

Fig. 2. - The overlap $q_{0}$ between relevant tours at temperature $T$ (see (4.5)). The curve for $T>0.6$ is obtained from the high temperature expansion, and then extrapolated linearly.

relevant tours, $P(L),(2.22)$ reduces in our replica symmetric case to

$$
P(L)=\frac{\beta^{2}}{2} \sum_{p=1}^{\infty}(-1)^{p-1} Q_{p}^{2} p^{2} \mathrm{e}^{-\beta p L}
$$

so that

$$
q_{0}=\int_{0}^{\infty} P(L) \mathrm{d} L-\frac{P(L=0)}{\beta}
$$


the first term is equal to one (this can be used as a check of the high temperature expansion of the $Q_{p}$ 's), so $q_{0}$ starts linearly from one at low temperatures if $P(L=0) \neq 0$.

Let us now compare these results with existing numerical data. The prediction (4.3) for the length of the shortest path seems to be in rather good agreement with the result of Kirkpatrick and Toulouse [7]. Indeed from their figure 1 one can plot the length versus $1 / N$. This is done in figure 3 . If one discards the data for $N>48$ in which presumably the ground state has not been found, one finds a nearly linear curve which extrapolates at $N=\infty$ to $E \sim 2.09$. As for the temperature dependence of the length it exhibits

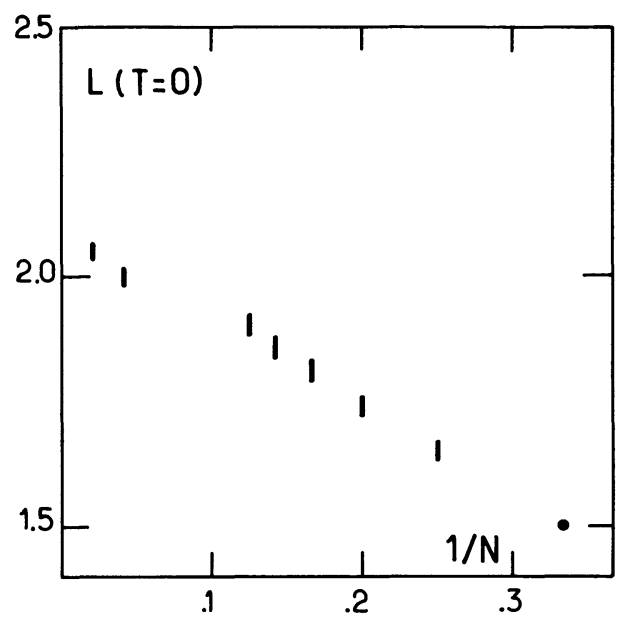

Fig. 3. - Plot of the optimal tour length obtained by Kirkpatrick and Toulouse [7] versus $1 / N$. typical features, such as the rather narrow freezing region around $T \sim 0.9$, which are also found numerically in [7]. Unfortunately the lack of statistics of [7] in the interesting low temperature range prevents from a really quantitative comparison.

From the theoretical point of view, we want to emphasize that we made two strong hypotheses on the solution $Q_{a_{1} \ldots a_{p}}^{\alpha_{1} \ldots a_{p}}$ of the saddle point equations. The first one is the spontaneous breaking of the rotational symmetry. This seems rather reasonable although we have neglected in this process some potential transverse freezing which is known to occur in vector spin glasses [17].

The second one is the approximation of replica symmetry. This may well be wrong if there is a breaking of ergodicity in this problem. As it stands it is very difficult to study the stability of our solution [18], so the simplest thing one could do would be to analyse precisely the relaxation times and the shape of $P(q)$ in this system with a Monte Carlo at finite temperature.

The length of the ground state is known to be rather well approximated by the replica symmetric ansatz (in the S.K. model it predicts an energy which is too low by $5 \%$ ). This might explain the success of [4-3], but clearly more precise numerical data and some theoretical predictions for the case of broken replica symmetry will be welcome.

\section{Acknowledgments.}

We are grateful to Scott Kirkpatrick for communicating us some details of his data.

\section{Appendix I.}

In this appendix we want to prove that in the TSP limit $(m \rightarrow 0$ and $\gamma \rightarrow \infty)$ for large values of $N$, the leading contributions arise from the terms in equation (2.10) where all replica indices are different.

Indeed for large $N$ we have

$$
\left(\sum_{i} S_{i, a_{1}}^{\alpha_{1}} \ldots S_{i, a_{p}}^{\alpha_{p}}\right)^{2} \simeq \sum_{i \neq j} S_{i, a_{1}}^{\alpha_{1}} S_{j, a_{1}}^{\alpha_{1}} \ldots S_{i, a_{p}}^{\alpha_{p}} S_{j, a_{p}}^{\alpha_{p}}
$$

where the terms with $i=j$ give a negligible contribution (proportional to $1 / N$ ).

It is convenient to evaluate equation (2.10) using a diagrammatic high temperature expansion, i.e. by expanding the exponential in powers of its argument. The diagrammatical rules are a simple generalization of the usual rules; we place a «link in replica $a_{k}$ " between sites $i$ and $j$ whenever the term

$$
S_{i, a_{1}}^{\alpha_{k}} S_{j, a_{k}}^{\alpha_{k}}
$$

is present.

The integration over $\mathrm{d} \mu\left(\mathbf{S}_{i, a}\right)$ implies that on each site $i$, for each replica $a$, there must be exactly an even number of identical spin components $S_{i, a}^{\alpha}$. Hence the diagrams which contribute have the following properties for each replica index $a$, there are only closed paths of « links in replica $a$ » and if in each replica $a$ there are $k_{a}$ closed paths, the weight of the diagram is

$$
m^{\Sigma k_{a}} \text {. }
$$

In the limit $\gamma \rightarrow \infty$, we get a factor $\gamma^{n N}$ only if all the sites in all the replicas will be visited by some path; 
$k_{a}$ must be different from zero for all values of $a$ : the leading contribution (in $m$ ) is given when

$$
k_{a}=1 \quad \forall a,
$$

(in this case a prefactor $m^{n}$ is produced).

Having established these results we can prove the main issue of the appendix. Each time that two replica indices are equal in the expression (AI.1) (say $a_{1}=a_{2}$ ), there is in the replica $a_{1}$ a small closed path between i and $\mathrm{j}$; if all the sites in the replica 1 must be visited, we must have $k_{1}>1$ and this term does not contribute in the TSP limit, as stated in the text.

\section{Appendix II.}

In this appendix we establish the formula for the distribution of overlaps $P(q)$. For a given sample of the lengths $l_{i j}$, we introduce two systems of $m$ component spins $\mathbf{S}_{i}$ and $\mathbf{S}_{i}^{\prime}$, and compute :

$$
\Xi\left(x_{i j}\right)=\int \prod_{i=1}^{N}\left[\mathrm{~d} \mu\left(\mathbf{S}_{i}\right) \mathrm{d} \mu\left(\mathbf{S}_{i}^{\prime}\right)\right] \exp \left[\gamma \sum_{i<j} x_{i j} \mathrm{e}^{-\beta N \frac{1}{r+1} l_{i j}}\left(\mathbf{S}_{i} \cdot \mathbf{S}_{j}+\mathbf{S}_{i}^{\prime} \cdot \mathbf{S}_{j}^{\prime}\right)\right]
$$

where $x_{i j}$ are bookkeeping auxiliary link variables which can be chosen for instance as independent random variables with a distribution $P(x)$ such that :

$$
\int P(x) \mathrm{d} x=1 ; \int P(x) x \mathrm{~d} x=1 ; \int P(x) x^{2} \mathrm{~d} x=1+\frac{y}{N} .
$$

In the TSP limit one has

$$
\Xi_{\mathrm{TSP}}\left(x_{i j}\right) \equiv \lim _{m \rightarrow 0} \frac{1}{m^{2}}\left[\lim _{\gamma \rightarrow \infty} \frac{1}{\gamma^{2 N}} \Xi\left(x_{i j}\right)\right]=\frac{1}{Z_{\mathrm{TSP}}^{2}} \sum_{t, t^{\prime}} \exp \left[-\beta N^{\frac{1}{r+1}}\left(L_{t}+L_{t^{\prime}}\right)\right] \prod_{(i, j) \in t} x_{i j} \prod_{\left(i^{\prime}, j^{\prime}\right) \in t^{\prime}} x_{i^{\prime} j^{\prime}} .
$$

Averaging over the $x$ variables with the distribution (AII .2) clearly gives a factor $\left(1+\frac{y}{N}\right)$ for each link common to the two tours $t$ and $t^{\prime}$, so that

$$
\int \prod_{i<j} P\left(x_{i j}\right) \mathrm{d} x_{i j} \Xi_{\mathrm{TSP}}\left(x_{i j}\right)=\sum_{t, t^{\prime}} \frac{\exp \left[-\beta N^{\frac{1}{r+1}}\left(L_{t}+L_{t^{\prime}}\right)\right]}{Z_{\mathrm{TSP}}^{2}} \mathrm{e}^{y q^{t} t}
$$

In order to compute the average over the distribution of the lengths in $(2.13)$ with the correct denominator $Z_{\mathrm{TSP}}^{2}$, one can use as before the replica method. After some work one gets for the characteristic function of $P(q)$ defined in (2.13):

$$
\begin{aligned}
& g(y)=\lim _{n \rightarrow 0} \frac{1}{n(n-1)} \sum_{a \neq b} \int \prod_{i<j} P\left(x_{i j}\right) \mathrm{d} x_{i j} \lim _{m \rightarrow 0} \frac{1}{m^{n}} \lim _{\gamma \rightarrow \infty} \frac{1}{\gamma^{n N}} \int \prod_{i=1}^{N} \prod_{c=1}^{n} \mathrm{~d} \mu\left(\mathbf{S}_{i, c}\right) \times \\
& \times \prod_{i<j} \exp \left[\gamma \mathrm{e}^{-\beta N \frac{1}{r+1} l_{i j}} \sum_{c} Z_{i j}^{c} \mathbf{S}_{i, c} \cdot \mathbf{S}_{j, c}\right]
\end{aligned}
$$

where $Z_{i j}^{c}=x_{i j}$ if $c=a$ or $b, Z_{i j}^{c}=1$ otherwise. The averages over the distributions of $l_{i j}$ and $x_{i j}$ for one link, using (2.8), gives :

$$
1+\frac{1}{N} \sum_{p=1}^{\infty} \frac{g_{p}}{p !} \gamma^{p}\left[\left(\sum_{c} \mathbf{S}_{i, c} \cdot \mathbf{S}_{j, c}\right)^{p}+\frac{y}{N} p(p-1)\left(\sum_{c} \mathbf{S}_{i, c} \cdot \mathbf{S}_{j, c}\right)^{p-2} \mathbf{S}_{i, a} \cdot \mathbf{S}_{j, a} \mathbf{S}_{i, b} \cdot \mathbf{S}_{j, b}\right]
$$

so that :

$$
\begin{array}{r}
g(y)=\lim _{n \rightarrow 0} \frac{1}{n(n-1)} \sum_{a \neq b} \lim _{m \rightarrow 0} \frac{1}{m^{n}} \lim _{\gamma \rightarrow \infty} \frac{1}{\gamma^{n N}} \int_{i=1} \prod_{a=1}^{N} \mathrm{~d} \mu\left(\mathbf{S}_{i, a}\right) \exp \frac{1}{2 N} \sum_{p=1}^{\infty} \frac{\gamma^{p} g_{p}}{p !} \sum_{\substack{a_{1} \ldots a_{p} \\
\alpha_{1} \ldots \alpha_{p}}}\left(\sum_{i} S_{i, a_{1}}^{\alpha_{1}} \ldots S_{i, a_{p}}^{\alpha_{p}}\right)^{2} \times \\
\exp \frac{y}{2 N^{2}} \sum_{p=1}^{\infty} \frac{\gamma^{p} g_{p}}{p !} p(p-1) \sum_{\substack{a_{3} \ldots a_{p} \\
\alpha_{3} \ldots a_{p}}}\left(\sum_{i} S_{i, a}^{\alpha} S_{i, b}^{\beta} S_{i, a_{3}}^{\alpha_{3}} S_{i, a_{p}}^{\alpha_{p}}\right)^{\alpha_{p}}
\end{array}
$$

Clearly in the first exponential factor the sites can be decoupled by a Gaussian transformation as in $(2.10)(2.11)$, 
while the second exponential is a term of relative order $1 / N$ which doesn't change the saddle point values of the variables. $Q_{a_{1} \ldots a_{p}}^{\alpha_{1} \ldots \alpha_{p}}$.

Using the saddle point equations to express this second exponential in terms of the $Q$ 's one gets :

$$
g(y)=\lim _{n \rightarrow 0} \frac{1}{n(n-1)} \sum_{a \neq b} \exp \frac{y}{2} \sum_{p=2}^{\infty} \frac{1}{p ! g_{p}} p(p-1) \sum_{\substack{a_{3} \ldots a_{p} \\ \neq a, b}}^{\sum_{\alpha_{3} \ldots \alpha_{p}}} \sum_{\alpha, \beta}\left(Q_{a b a_{3} \ldots a_{p}}^{\alpha \beta \alpha_{3} \ldots \alpha_{p}}\right)^{2}
$$

from which the formula (2.14) for $P(q)$ follows immediately.

\section{Appendix III.}

We compute the one-site partition function in the replica symmetric ansatz, $z$, given in (3.4). The quantity $\sum_{a_{1} \ldots a_{p}}^{\prime} X_{a_{1}} \ldots X_{a_{p}}$ (where $X_{a}=\mathbf{S}^{a} \cdot \mathbf{u}$ ) differs from $\left(\sum_{a} X_{a}\right)^{p}$ by terms with equal indices, involving sums of the type $\sum_{a} X_{a}^{2}, \sum_{a} X_{a}^{3}, \ldots$ However from (2.5) the integral over $\mathrm{d} \mu\left(\mathbf{S}_{a}\right)$ of a quantity involving at least three spins with the same replica index, $a$, vanishes in the $m \rightarrow 0$ limit. Hence one can forget about the terms $\sum_{a} X_{a}^{k}, k \geqslant 3$ in going from $\sum_{a_{1} \ldots a_{p}}^{\prime} X_{a_{1}} \ldots X_{a_{p}}$ to $\sum_{a_{1} \ldots a_{p}} X_{a_{1}} \ldots X_{a_{p}}$. We write

$$
\sum_{a_{1} \ldots a_{p}}^{\prime} X_{a_{1}} \ldots X_{a_{p}}=\left(\sum_{a} X_{a}^{2}\right)^{p / 2} Q_{p}\left(\frac{\sum_{a} X_{a}}{\sqrt{\sum_{a} X_{a}^{2}}}\right)
$$

where $Q_{p}(x)$ is a polynomial of degree $p: Q_{p}=X^{p}+q_{p, 2} X^{p-2}+\cdots$. From the recurrence relation

$$
\sum_{a_{1} \ldots a_{p}}^{\prime} X_{a_{1}} \ldots X_{a_{p}}=\left(\sum_{a_{1} \ldots a_{p-1}}^{\prime} X_{a_{1}} \ldots X_{a_{p-1}}\right)\left(\sum_{b} X_{b}\right)-(p-1)\left(\sum_{b} X_{b}^{2}\right)\left(\sum_{a_{1} \ldots a_{p-2}}^{\prime} X_{a_{1}} \ldots X_{a_{p-2}}\right)
$$

one finds that $Q_{p}(X)$ is nothing but the Hermite polynomial $H e_{p}(X)[19]$ :

$$
\begin{aligned}
\sum_{a_{1} \ldots a_{p}}^{\prime} X_{a_{1}} \ldots X_{a_{p}} & =\left(\sum_{a} X_{a}^{2}\right)^{p / 2} H e_{p}\left(\frac{\sum_{a} X_{a}}{\sqrt{\sum_{a} X_{a}^{2}}}\right) \\
& =\int_{-\infty}^{+\infty} D t\left[\sum_{a} X_{a}+i t \sqrt{\sum_{a} X_{a}^{2}}\right]^{p}
\end{aligned}
$$

where

$$
D t=\frac{d t}{\sqrt{2 \pi}} \mathrm{e}^{-t^{2} / 2} .
$$

The presence of Hermite polynomials is not surprising : indeed the subtraction of terms with the same replica index leeds to a combinatorial problem which is the same as that of finding the normal product : $\varphi^{p}:$ of a free field $\varphi$. In this case the result is well known

$$
: \varphi^{p}:=\left\langle\varphi^{2}\right\rangle^{p / 2} H e_{p}\left(\frac{\varphi}{\sqrt{\left\langle\varphi^{2}\right\rangle}}\right)
$$

So the partition function $z$ is

$$
z=\int \prod_{a} \mathrm{~d} \mu\left(\mathbf{S}_{a}\right) \exp \left\{\sum_{p=1}^{\infty} \frac{\gamma^{p / 2}}{p !} Q_{p} \int D t\left[\sum_{a} \mathbf{S}_{a} \cdot \mathbf{u}+i t \sqrt{\sum_{a}\left(\mathbf{S}_{a} \cdot \mathbf{u}\right)^{2}}\right]^{p}\right\} .
$$

We write $X=\sqrt{\gamma} \sum_{a} \mathbf{S}_{a} \cdot \mathbf{u}, Y=\gamma \sum_{a}\left(\mathbf{S}_{a} \cdot \mathbf{u}\right)^{2}$, and we impose these constraints through $\delta$ functions written 
in integral representation

$$
\begin{aligned}
z=\int_{-\infty}^{+\infty} \frac{\mathrm{d} \hat{X}}{2 \pi} \int_{-\infty}^{+\infty} \mathrm{d} X \int_{-\infty}^{+\infty} \frac{\mathrm{d} \hat{Y}}{2 \pi} \int_{-\infty}^{+\infty} \mathrm{d} Y \mathrm{e}^{-i X X-i Y Y} \times \\
\quad \times \exp \left\{\sum_{p=1}^{\infty} \frac{Q_{p}}{p !} \int D t[X+i t \sqrt{Y}]^{p}\right\} \prod_{a}\left[\int \mathrm{d} \mu\left(\mathbf{S}_{a}\right) \mathrm{e}^{i \hat{\mathbf{X}} / \bar{\gamma} \mathbf{s}_{a \cdot \mathbf{u}}+i \hat{\mathbf{Y}} /\left(\mathbf{S}_{a} \cdot \mathbf{u}\right)^{2}}\right] .
\end{aligned}
$$

The integral over the $\mathbf{S}^{a}$, in the limit $m \rightarrow 0$, is

$$
\left\{1+\gamma\left[\frac{(i \hat{\mathbf{X}})^{2}}{2}+i \hat{\mathbf{Y}}\right]\right\}^{n}
$$

In the TSP limit we are interested in the term of the order of $\gamma^{n}$ of $z$ which is

$$
z \sim \gamma^{n}\left\{\left.\left[\frac{1}{2} \frac{\partial^{2}}{\partial X^{2}}+\frac{\partial}{\partial Y}\right]^{n} \exp \left[\sum_{p=1}^{\infty} \frac{Q_{p}}{p !} \int D t(X+i t \sqrt{Y})^{p}\right]\right|_{X=Y=0}\right\} .
$$

Having extracted the $\gamma^{n}$ term we can go on and take the limit $n \rightarrow 0$. One way to do it is to write

$$
\frac{z}{\gamma^{n}}=\left.\left(\frac{\partial}{\partial \lambda}\right)^{n} \exp \lambda\left[\frac{1}{2} \frac{\partial^{2}}{\partial X^{2}}+\frac{\partial}{\partial Y}\right] \exp \left[\sum_{p=1}^{\infty} \frac{Q_{p}}{p !} \int D t[X+i t \sqrt{Y}]^{p}\right]\right|_{\lambda=0}
$$

where

$$
f(\lambda)=\int D z \exp \left\{\sum_{p=1}^{\infty} \frac{Q_{p}}{p !} \sqrt{\lambda}^{p} H e_{p}(z)\right\}
$$

is a function of $\lambda$ with $f(0)=1$, so that

$$
\gamma^{-n} z=\left.\left(\frac{\partial}{\partial \lambda}\right)^{n} f(\lambda)\right|_{\lambda=0} \sim 1+n \int_{0}^{\infty} \frac{\mathrm{d} \lambda}{\lambda}\left[\mathrm{e}^{-\lambda}-\int D z \exp \left(\sum_{p=1}^{\infty} \frac{Q_{p}}{p !}(i \sqrt{\lambda})^{p} H e_{p}(z)\right)\right] .
$$

\section{References}

[1] Kirkpatrick, S. and Sherrington, D., Phys. Rev. B 17 (1978) 4384.

[2] Barahona, F., J. Phys. A 15 (1982) 3241.

[3] Garey, M. R. and Johnson, D. S., Computers and Intractability (Freeman, San Francisco) 1979.

[4] KIRKPATRICK, S., Lecture Notes in Physics 149 (Springer, Berlin) 1981, p. 280 ;

KirkPatrick, S., Gelatt, C. D. Jr., Vecchi, M. P., Science 220 (1983) 671.

[5] Siarry, P. and Dreyfus, G., J. Physique Lett. 45 (1984) L-39.

[6] Bonomi, E. and Lutton, J. L., SIAM Rev. 26 (1984) 551.

[7] Kirkpatrick, S. and Toulouse, G., J. Physique 46 (1985) 1277.

[8] Parisi, G., J. Phys. A 13 (1980) L-115, 1101, 1887.

[9] Mézard, M., Parisi, G., Sourlas, N., Toulouse, G. and Virasoro, M. A., Phys. Rev. Lett. 52 (1984) 1156, and J. Physique 45 (1984) 843.
[10] Mézard, M. and Virasoro, M. A., J. Physique 46 (1985) 1293.

[11] Ḿ́zard, M. and Parisi, G., J. Physique Lett. 46 (1985) L-771.

[12] Fu, Y. and Anderson, P. W., Princeton University preprint (1985).

[13] Vannimenus, J. and Mézard, M., J. Physique Lett. 45 (1984) L-1145.

[14] De Gennes, P. G., Phys. Lett. A 38 (1972) 339.

[15] ORland, H., J. Physique Lett. 46 (1985) L-763.

[16] Parisi, G., Phys. Rev. Lett. 50 (1983) 1946.

[17] Gabay, M. and Toulouse, G., Phys. Rev. Lett. 47 (1981) 201.

[18] What we mean is the analog of the computation for spin glasses done in DE Almeida, J. R. L. and Thouless, D. J., J. Phys. A 11 (1978) 983.

[19] Abramowitz, M. and Stegun, I. A. ed., Handbook of mathematical Functions, National Bureau of Standards. Applied Mathematics Series (1964). 